The Astrophysical Journal, 678:1298-1303, 2008 May 10

(C) 2008. The American Astronomical Society. All rights reserved. Printed in U.S.A.

\title{
THE FIRST MID-INFRARED SPECTRA OF COOL WHITE DWARFS
}

\author{
Mukremin Kilic, ${ }^{1}$ Piotr M. Kowalski, ${ }^{2}$ Fergal Mullally, ${ }^{3}$ William T. Reach, ${ }^{4}$ and Ted von Hippel ${ }^{5}$ \\ Received 2007 October 11; accepted 2007 December 13
}

\begin{abstract}
We present the first mid-infrared spectra of two cool white dwarfs obtained with the Spitzer Space Telescope. We also present 3.5-8 $\mu \mathrm{m}$ photometry for 19 cool white dwarfs with $5000 \mathrm{~K} \leq T_{\text {eff }} \leq 9000 \mathrm{~K}$. We perform a detailed model atmosphere analysis of these white dwarfs by fitting their UBVRIJHK and Spitzer photometry with state-ofthe-art model atmospheres, and demonstrate that the optical and infrared spectral energy distributions of cool white dwarfs are well reproduced by our grid of models. Our mid-infrared photometry and $7.5-14.5 \mu \mathrm{m}$ spectrum of WD 0018-267 are consistent with a $T_{\text {eff }}=5720 \mathrm{~K}$, pure hydrogen white dwarf model atmosphere. On the other hand, LHS 1126 remains peculiar, with significant mid-infrared flux deficits in all IRAC bands and a featureless spectrum in the 5.2-7.5 $\mu \mathrm{m}$ range. Even though this deficit is attributed to collision-induced absorption (CIA) due to molecular hydrogen, the shape of the deficit cannot be explained with current CIA opacity calculations. The infrared portion of the LHS 1126 spectral energy distribution is best fit with a power-law index of -1.99 , identical to a Rayleigh-Jeans spectrum. This argues that the deficit may be due to an unrecognized graylike opacity source in the infrared.
\end{abstract}

Subject headings: infrared: stars — stars: individual (WD 0018-267, WD 0038-226, LHS 1126) — white dwarfs

\section{INTRODUCTION}

Cool white dwarfs (WDs) come in three different flavors: $\mathrm{H}$-dominated, He-dominated, and mixed $\mathrm{H} / \mathrm{He}$ atmospheres. The atmospheric composition for WDs hotter than about $11,000 \mathrm{~K}$ can be easily identified from the observed hydrogen and helium lines. However, helium lines disappear below 11,000 K, and hydrogen becomes invisible below $5000 \mathrm{~K}$. Optical spectroscopy cannot differentiate between the three types of atmospheric compositions for WDs cooler than $5000 \mathrm{~K}$. The dominant opacity sources in cool H-rich WD atmospheres are believed to be collisioninduced absorption (CIA) in the infrared (Hansen 1998; Saumon \& Jacobson 1999) and Ly $\alpha$ in the ultraviolet (Kowalski \& Saumon 2006; Koester \& Wolff 2000). CIA opacity is strongly wavelengthdependent and is expected to produce molecular absorption features in the near-infrared (Frommhold 1993). For very cool WDs, these features extend into the optical region (Harris et al. 2001; Gates 2004).

The primary opacity source in He-rich WDs is $\mathrm{He}^{-}$free-free absorption (Kowalski et al. 2007). In mixed H/He atmospheres, collisions between $\mathrm{H}_{2}$ molecules and neutral helium can induce CIA. Helium atmospheres are much denser than the hydrogen atmospheres at the same temperature; therefore, CIA becomes significant at higher temperatures compared to pure hydrogen atmospheres. The only way to differentiate between pure $\mathrm{H}$, pure $\mathrm{He}$, and mixed $\mathrm{H} / \mathrm{He}$ composition is to perform a detailed model atmosphere analysis of both the optical and the infrared colors of cool WDs.

Cool WDs define the faint end of the WD luminosity function, and they are used to determine the ages of the Galactic disk and halo, and nearby globular clusters (Winget et al. 1987; Liebert et al. 1988; Harris et al. 2006; Hansen et al. 2007). Due to differ-

\footnotetext{
1 Department of Astronomy, The Ohio State University, Columbus, OH 43210; kilic@astronomy.ohio-state.edu.

2 Lehrstuhl für Theoretische Chemie, Ruhr-Universität Bochum, 44780 Bochum, Germany.

3 Department of Astrophysical Sciences, Princeton University, Princeton, NJ 08544.

4 Spitzer Science Center, California Institute of Technology, Pasadena, CA 91125.

5 Department of Astronomy, University of Texas, Austin, TX 78712.
}

ent opacities, the atmospheric composition affects the WD cooling rate. Hence, determining an accurate temperature, luminosity, and age for cool WDs requires determining the atmospheric compositions of the stars. Bergeron et al. (2001) successfully fit the optical and near-infrared colors of a sample of nearby WDs, and they could explain the cool WD spectral energy distributions (SEDs) by different atmospheric compositions. They found that the coolest WDs in the solar neighborhood have $T_{\text {eff }}=4200 \mathrm{~K}$, corresponding to an age of 8 Gyr.

Kilic et al. (2006) presented Spitzer 4.5 and $8 \mu \mathrm{m}$ photometry of 18 cool WDs, including 14 WDs from the Bergeron et al. (2001) sample. Their preliminary analysis showed that several WDs with $T_{\text {eff }}<6000 \mathrm{~K}$ were slightly fainter than expected in the mid-infrared. In addition, they found that LHS 1126, a WD believed to have a mixed $\mathrm{H} / \mathrm{He}$ atmosphere, displayed strong flux deficits in the 4.5 and $8 \mu \mathrm{m}$ bands. Fainter mid-infrared fluxes implied that the bolometric corrections for cool WDs may be wrong, and the age estimates may be affected as well. Current CIA calculations do not predict absorption bands in the midinfrared, which would imply that either the CIA calculations in WD atmospheres are incomplete, or these flux deficits are caused by some other mechanism.

Tremblay \& Bergeron (2007) reanalyzed the Spitzer photometry for 12 of the WDs presented in Kilic et al. (2006), and with the exception of LHS 1126, they did not find any significant flux deficit at low effective temperatures. In order to understand the mid-infrared SEDs of cool WDs and the CIA opacity, we used the Infrared Spectrograph (IRS) on the Spitzer Space Telescope to observe two nearby, relatively bright cool white dwarfs, WD 0018-267 and LHS $1126 .{ }^{6}$ In this paper we present our mid-infrared spectroscopy of these two WDs and new Infrared Array Camera (IRAC) photometry of several WDs. In order

${ }^{6}$ Mid-infrared spectroscopy of two WDs (G29-38 and GD 362) with $T_{\text {eff }}=$ $(1-1.2) \times 10^{4} \mathrm{~K}$ was presented by Reach et al. (2005a) and Jura et al. (2007). However, both of these stars have circumstellar debris disks, and their IRS spectra are dominated by the emission from the debris disks. Hence, these spectra cannot be used to constrain the mid-infrared SEDs of cool WDs. In addition, our two targets are about $5000 \mathrm{~K}$ cooler than GD 362, which corresponds to an age difference of about 3 Gyr. Therefore, our IRS spectra of WD 0018-267 and LHS 1126 are the first mid-infrared spectra of cool WD photospheres. 
TABLE 1

Infrared Photometry of Cool White Dwarfs

\begin{tabular}{|c|c|c|c|c|c|c|}
\hline Object & Spectral Type & $\begin{array}{l}T_{\text {eff }} \\
(\mathrm{K})\end{array}$ & $\begin{array}{c}F_{3.6 \mu \mathrm{m}} \\
(\mathrm{mJy})\end{array}$ & $\begin{array}{c}F_{4.5 \mu \mathrm{m}} \\
(\mathrm{mJy})\end{array}$ & $\begin{array}{c}F_{5.8 \mu \mathrm{m}} \\
(\mathrm{mJy})\end{array}$ & $\begin{array}{c}F_{8.0 \mu \mathrm{m}} \\
(\mathrm{mJy})\end{array}$ \\
\hline WD $0009+501 \ldots \ldots \ldots \ldots \ldots \ldots$ & DAP & 6631 & & $0.9562 \pm 0.0514$ & & $0.3159 \pm 0.0186$ \\
\hline 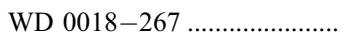 & DA & 5720 & $4.4218 \pm 0.1396$ & $2.8525 \pm 0.0956$ & $1.9086 \pm 0.0699$ & $1.0174 \pm 0.0435$ \\
\hline 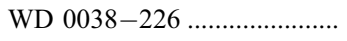 & $\mathrm{C}_{2} \mathrm{H}:$ & 5400: & $0.7996 \pm 0.0307$ & $0.4943 \pm 0.0162$ & $0.3068 \pm 0.0317$ & $0.1953 \pm 0.0319$ \\
\hline 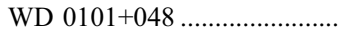 & DA & 8360 & $\ldots$ & $0.7693 \pm 0.0264$ & $\ldots$ & $0.2808 \pm 0.0307$ \\
\hline WD $0126+101 \ldots \ldots \ldots \ldots \ldots \ldots \ldots$ & DA & 8926 & $\ldots$ & $0.4592 \pm 0.0214$ & $\ldots$ & $0.1364 \pm 0.0668$ \\
\hline WD $0141-675 \ldots \ldots \ldots \ldots \ldots \ldots \ldots$ & DA & 6577 & $2.5736 \pm 0.0810$ & $1.6736 \pm 0.0569$ & $1.0423 \pm 0.0503$ & $0.5788 \pm 0.0246$ \\
\hline WD $0552-041$ & $\mathrm{DZ}$ & 5509 & $\ldots$ & $1.8454 \pm 0.0660$ & $\ldots$ & $0.7383 \pm 0.0666$ \\
\hline WD $0553+053$ & DAP & 5869 & $\ldots$ & $1.6701 \pm 0.0518$ & $\ldots$ & $0.5185 \pm 0.0536$ \\
\hline 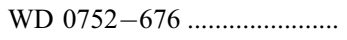 & DA & 5661 & $\ldots$ & $2.1580 \pm 0.0770$ & $\ldots$ & $0.7710 \pm 0.0379$ \\
\hline WD 0839-327 ….................... & DA & 8797 & $\ldots$ & $4.0963 \pm 0.1409$ & $\ldots$ & $1.4482 \pm 0.0559$ \\
\hline 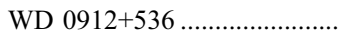 & DCP & 7660 & $\ldots$ & $1.1166 \pm 0.0356$ & $\ldots$ & $0.4023 \pm 0.0330$ \\
\hline WD $1055-072$....................... & $\mathrm{DC}$ & 7740 & $\ldots$ & $0.6567 \pm 0.0249$ & $\ldots$ & $0.2512 \pm 0.0356$ \\
\hline WD $1121+216 \ldots \ldots \ldots \ldots \ldots \ldots$ & DA & 7328 & $\ldots$ & $0.8229 \pm 0.0302$ & $\ldots$ & $0.3294 \pm 0.0185$ \\
\hline 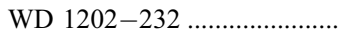 & DA & 9115 & $\ldots$ & $1.9858 \pm 0.0692$ & $\ldots$ & $0.6835 \pm 0.0358$ \\
\hline WD $1223-659$........................ & DA & 7826 & $\ldots$ & $0.9217 \pm 0.0438$ & $\ldots$ & $0.3529 \pm 0.0690$ \\
\hline 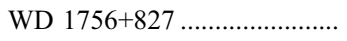 & DA & 7259 & $\ldots$ & $0.7614 \pm 0.0237$ & $\ldots$ & $0.2601 \pm 0.0280$ \\
\hline 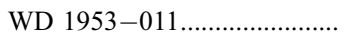 & DAP & 7894 & $\ldots$ & $1.1540 \pm 0.0425$ & $\ldots$ & $0.4395 \pm 0.0263$ \\
\hline 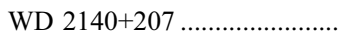 & DQ & 9700 & $1.8462 \pm 0.0632$ & $1.2033 \pm 0.0397$ & $0.7877 \pm 0.0482$ & $0.3948 \pm 0.0143$ \\
\hline WD 2359-434 …......................... & DA & 8633 & $\ldots$ & $1.7898 \pm 0.0583$ & $\ldots$ & $0.6075 \pm 0.0636$ \\
\hline
\end{tabular}

NotE.-The $8 \mu \mathrm{m}$ photometry of WD $1121+216$ is affected by a nearby star.

to resolve the discrepancy between the Kilic et al. and Tremblay $\&$ Bergeron analysis, we also reanalyze the photometry for 19 cool WDs. Our IRAC and IRS observations are discussed in $\S \S 2$ and 3 , respectively, while the implications of these data are discussed in $\S 4$.

\section{IRAC OBSERVATIONS}

Observations reported here were obtained as part of the Spitzer Cycle 1 GO-Program 2313 (PI: M. Kuchner) and Cycle 2 GOProgram 20026 (PI: T. von Hippel). As part of our Cycle 1 program, we obtained 4.5 and $8 \mu \mathrm{m}$ data for $18 \mathrm{cool}$ WDs that were presented in Kilic et al. (2006). Here we reanalyze those data with the latest Spitzer Science Center pipeline products. As part of our Cycle 2 program, we used IRAC (Fazio et al. 2004) to obtain 3.6 and $5.8 \mu \mathrm{m}$ photometry of four cool white dwarfs including WD 0018-267 and LHS 1126. An integration time of $30 \mathrm{~s}$ per dither, with five dithers for each target, was used. The data reduction procedures are similar to the procedures employed by Kilic et al. (2006). The only difference in our reduction procedures is the way we perform the array location-dependent corrections for the IRAC instrument. Kilic et al. (2006) multiplied the BCD images from the S11.1.0 pipeline with the location-dependent correction image before performing photometry. We now perform aperture photometry on the individual BCD frames from the S14.0.0 pipeline, and multiply the photometry by the coefficients obtained from the correction image supplied by the Spitzer Science Center. This small difference in our procedure resulted in $+2 \% \pm 0.7 \%$ and $-0.6 \% \pm 4 \%$ differences in our 4.5 and $8 \mu \mathrm{m}$ photometry, respectively. The photometric error bars were estimated from the observed scatter in the five images (corresponding to five dither positions) plus the $3 \%$ absolute calibration error, added in quadrature.

Most of our objects were observed by Bergeron et al. (2001) and therefore have accurate $B V R I$ photometry. For the remaining objects, we used $U B V$ photometry from the McCook \& Sion catalog. ${ }^{7}$ The near-infrared photometry comes from the 2 MASS

\footnotetext{
${ }^{7}$ Vizier Online Data Catalog, 3235 (G. P. McCook \& E. M. Sion, 2006).
}

All-Sky Point Source Catalog. ${ }^{8}$ We fit the UBVRIJHK and Spitzer photometry for our targets with the current state-of-the-art WD model atmospheres (including the missing opacity from the Ly $\alpha$ wing; Kowalski \& Saumon 2006) to determine $T_{\text {eff }}$ and $\log g$, if parallax measurements are available. Otherwise, we assume $\log g=8$. Our fitting procedure is the same as the method employed by Tremblay \& Bergeron (2007).

The average fluxes measured from the Spitzer images are given in Table 1. Figure 1 shows the optical and infrared SEDs (error bars) for 18 cool WDs with $T_{\text {eff }} \leq 9000 \mathrm{~K}$. The expected fluxes from synthetic photometry of WD model atmospheres are shown as open circles. This figure demonstrates that our grid of models reproduces the observed SEDs well in the entire range from 0.4 to $8 \mu \mathrm{m}$. The agreement is better for the Bergeron et al. (2001) stars, as the optical photometry is more accurate. We note that the $U$-band photometry is slightly discrepant for some stars (e.g., WD $0552-041$ is a DZ; hence, the $U$ - and $B$-band photometry is affected by metals). This is caused by either the uncertainties in the $U$-band observations obtained from the McCook \& Sion catalog, ${ }^{9}$ or the presence of charged species in the atmosphere which may change the predicted $U$-band fluxes. Stark broadening is not included in our $\operatorname{Ly} \alpha$ model that is constructed for neutral medium. Stark broadening becomes significant at $T_{\text {eff }} \geq$ $7000 \mathrm{~K}$, and hence the $U$-band flux for stars hotter than $7000 \overline{\mathrm{K}}$ may be overestimated in our models.

Figure 2 presents the ratio of observed to predicted 4.5 and $8 \mu \mathrm{m}$ fluxes for hydrogen (crosses) and helium (filled circles) dominated atmosphere WDs. Our photometric methods are consistent with those used for the IRAC absolute calibration (Reach et al. 2005b), and therefore should be accurate to $3 \%$. We note that the $8 \mu \mathrm{m}$ photometry of WD $1121+216$ is affected by a nearby star, and therefore it is not included in the bottom panel. The observed $4.5 \mu \mathrm{m}$ fluxes are completely consistent with the WD model predictions, showing that in general there are not atmospheric deviations of WDs in the mid-infrared. The $8 \mu \mathrm{m}$

\footnotetext{
${ }^{8}$ Vizier Online Data Catalog, 2246 (R. M. Cutri et al., 2003).

9 Vizier Online Data Catalog, 3235 (G. P. McCook \& E. M. Sion, 2006).
} 


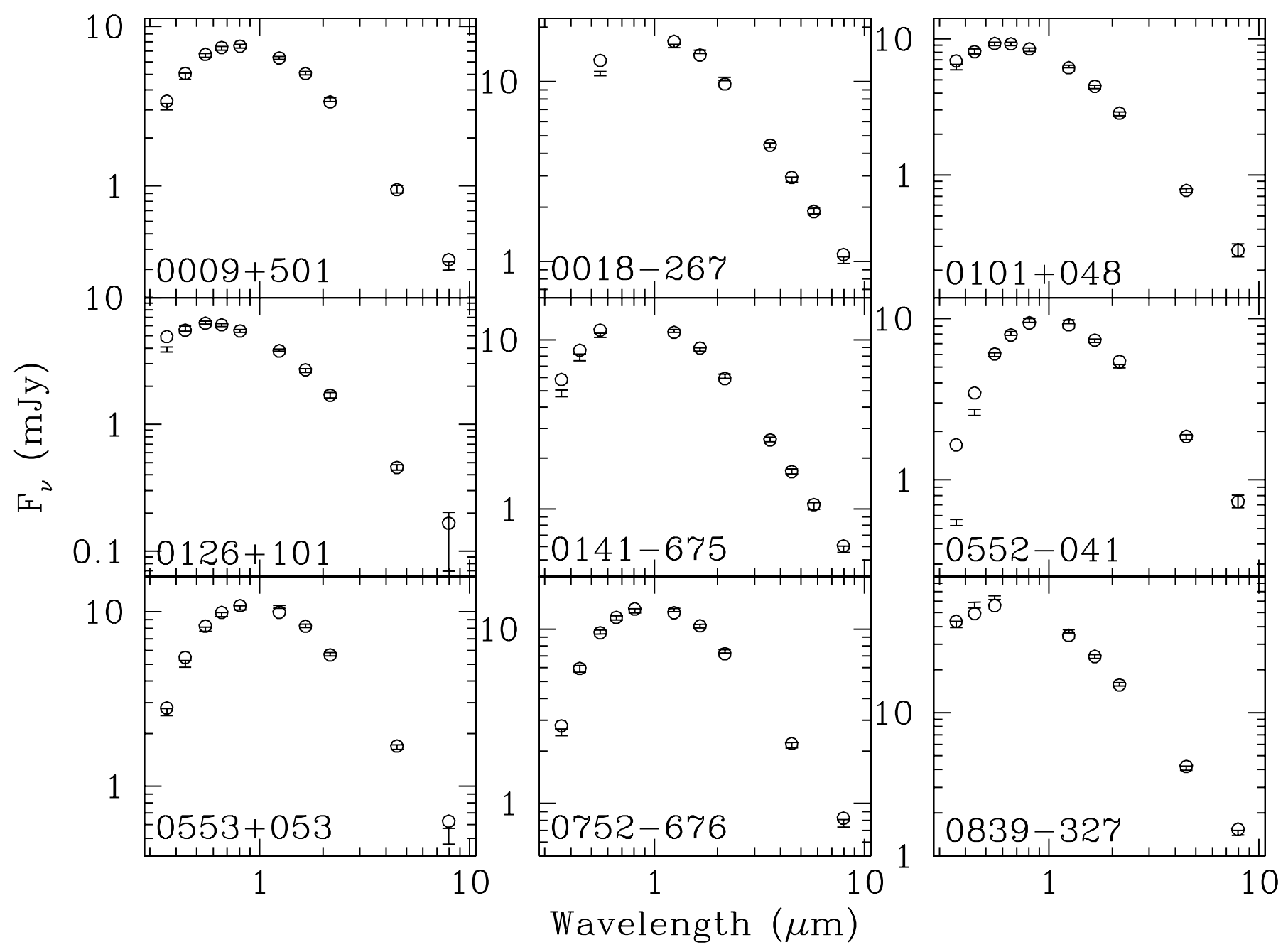

FIG. 1.-SEDs of cool WDs observed in our Spitzer Cycle 1 and Cycle 2 programs. The observed fluxes are shown as error bars, whereas the expected flux distributions from synthetic photometry of WD models are shown as open circles.

fluxes are on average fainter by $4 \%$ compared to expectations. However, given the $3 \%$ uncertainty in the IRAC absolute flux calibration, we conclude that the IRAC photometry of cool WDs is consistent with WD model atmospheres; as a corollary, WDs provide a calibration verification independent of the $\mathrm{A}$ and $\mathrm{K}$ stars that were included in the absolute calibration of IRAC.

Using the photometry from Kilic et al. (2006), Tremblay \& Bergeron (2007) found that the 12 WDs that they have analyzed were on average fainter by $2 \%$ in the $4.5 \mu \mathrm{m}$ band, whereas they could fit the $8 \mu \mathrm{m}$ photometry fairly well with their models. Our new photometry is $2 \%$ brighter in the $4.5 \mu \mathrm{m}$ band compared to Kilic et al. (2006). Therefore, our observations are consistent with the Tremblay \& Bergeron (2007) analysis as well.

Other Spitzer WD surveys also targeted several cool WDs. The DAZ survey of Debes et al. (2007) included four stars with $T_{\text {eff }} \sim$ $5300-8500 \mathrm{~K}$. The ratios of the observed fluxes to the predicted fluxes for these stars are $0.96 \pm 0.04$ and $0.98 \pm 0.05$ in the IRAC2 and IRAC4 bands, respectively. The metal-rich WD survey of Farihi et al. (2008) included seven stars with $T_{\text {eff }} \sim$ $5200-8000 \mathrm{~K}$ that have mid-IR flux distributions consistent with the models.

\section{IRS SPECTROSCOPY}

A better way to test the agreement between the WD models and the mid-infrared observations is to compare spectroscopy.
As part of our Cycle 2 GO-Program 20026 (PI: T. von Hippel), we used the Infrared Spectrograph (IRS; Houck et al. 2004) Short-Low module in the first order to obtain 7.4-14.5 $\mu \mathrm{m}$ spectroscopy of WD $0018-267$ with a resolving power of $\sim 90$. These observations consisted of 13 cycles of 4 minute exposures and were performed on 2006 July $121: 12$ UT. In addition, as part of our Cycle 3 GO-Program 30208 (PI: M. Kilic), we used the IRS Short-Low module in the second order to obtain 5.2-8.7 $\mu \mathrm{m}$ spectroscopy of LHS 1126. These observations consisted of 52 cycles of 1 minute exposures and were performed on 2007 August 202 : 48 UT. Both observations consisted a series of exposures obtained at two positions along the slit. The IRS images at each nod position were filtered such that all pixels deviating far from a 5 pixel wide median in the dispersion direction were replaced by that median. Then the nods were differenced and the spectra extracted using the Spitzer IRS Custom Extractor (SPICE) in "optimal" extraction mode. For LHS 1126 the first-minus-second nod difference was used, as the second-minus-first difference was contaminated over half the slit by stray light.

Figure 3 presents the weighted mean of the spectra for WD 0018-267 from the two nods (jagged line) compared to our bestfit model atmosphere fit (solid line) to the VJHK and IRAC1IRAC4 band photometry (error bars). Since the distance to the WD is not known, we assumed $\log g=8$. This spectrum shows that WD model atmospheres are able to explain the observed 


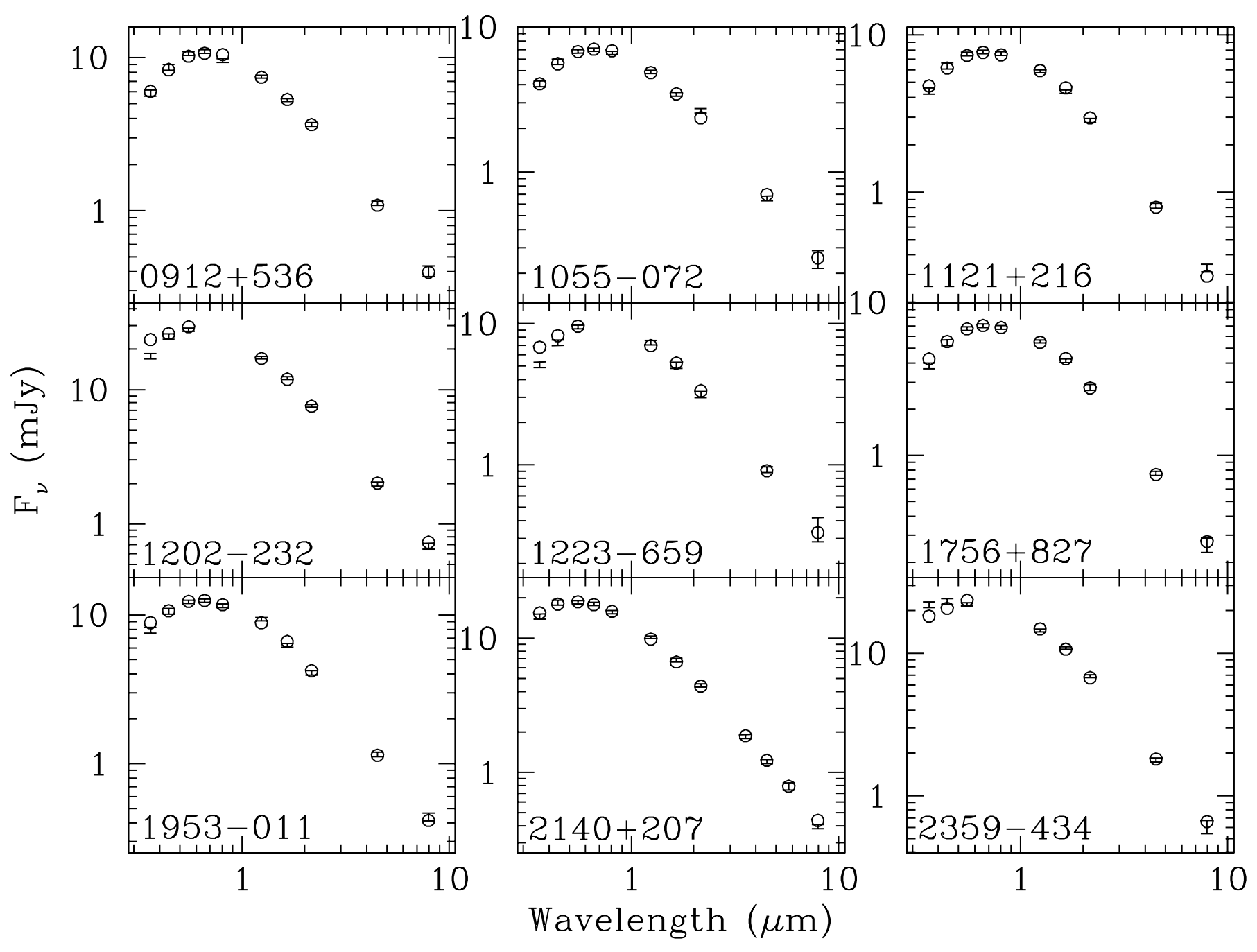

FIg. 1-Continued

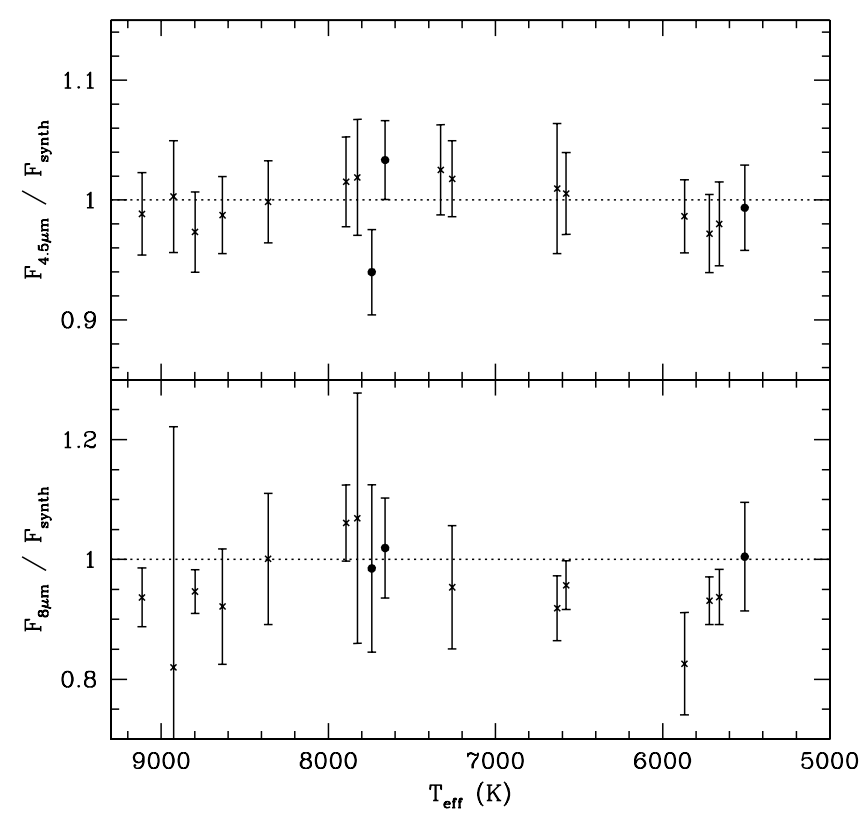

FIG. 2.-Ratio of observed to predicted Spitzer fluxes for $\mathrm{H}$ (crosses) and $\mathrm{He}$ ( filled circles) dominated atmosphere WDs. The predicted fluxes and $T_{\text {eff }}$ values are obtained from simultaneous fits to the UBVRIJHK and IRAC1-IRAC4 photometric data, where available.

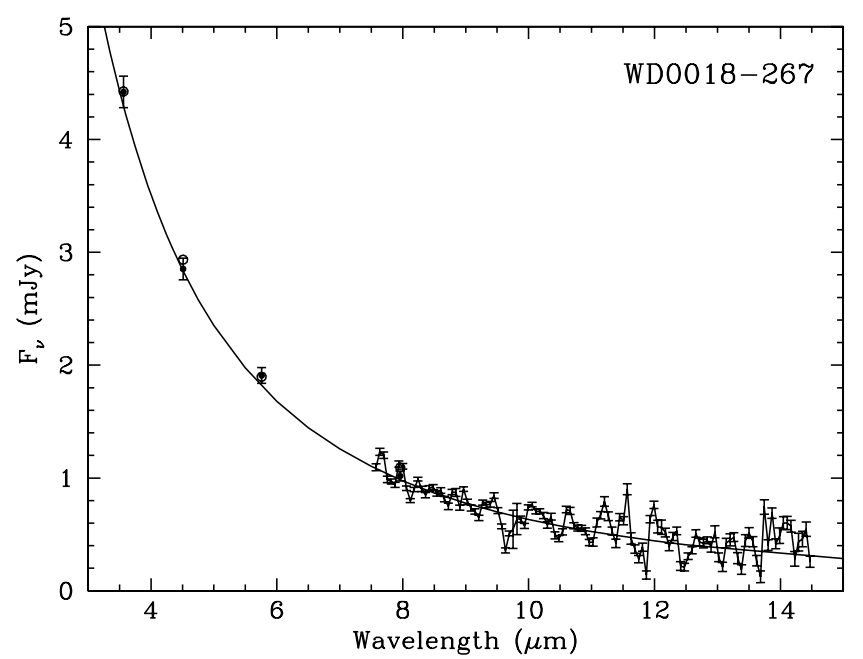

FIG. 3.- IRAC photometry ( filled circles with error bars) and IRS spectroscopy (error bars) of WD 0018-267 compared to the best-fitting pure $\mathrm{H}$ model atmosphere with $T_{\text {eff }}=5720 \mathrm{~K}$ (solid line). Open circles show the synthetic photometry of the best-fit model in the IRAC bands. 


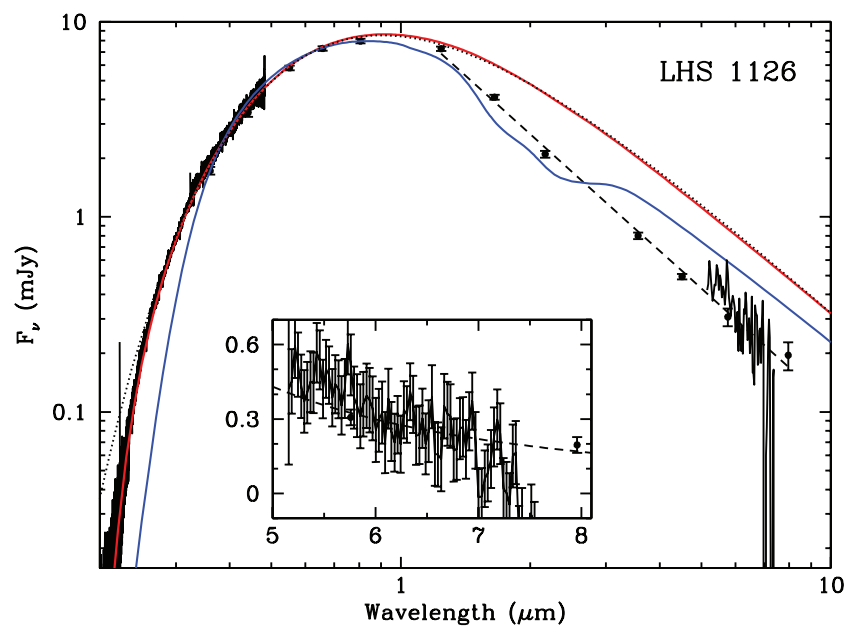

FIG. 4.- SED of LHS 1126, along with a $5400 \mathrm{~K}$ pure He atmosphere model (dotted line). Red and blue solid lines show the model atmospheres using the best-fit parameters from Wolff et al. [2002; $T_{\text {eff }}=5400 \mathrm{~K}, \log g=7.9$, and $\log (\mathrm{He} / \mathrm{H})=5.51]$ and Bergeron et al. $\left[1997 ; T_{\text {eff }}=5400 \mathrm{~K}, \log g=7.9\right.$, and $\log (\mathrm{He} / \mathrm{H})=1.86]$, respectively. The dashed line shows a least-squares fit to the infrared photometry data only. The infrared portion of the SED is best fit with a power-law index of -1.99 . The inset shows the $5-8 \mu \mathrm{m}$ spectral region in detail.

SED of a $5700 \mathrm{~K}$ pure hydrogen atmosphere WD fairly well. We note that we did not use the IRS spectrum in our model fits. In addition, we did not normalize the spectrum to match the IRAC photometry or the expected SED from the model atmospheres.

Figure 4 shows the ultraviolet, optical, and infrared SED of LHS 1126 including our new IRS spectrum. A remarkable feature of this SED is that the optical portion can be fit with a $\sim 5400 \mathrm{~K}$ WD model, whereas the infrared flux is deficient, and it gradually decreases with no observable molecular features. Red and blue solid lines show the WD model atmospheres assuming atmospheric parameters derived by Wolff et al. (2002) and Bergeron et al. (1997), respectively. These models include all nonideal physics of dense helium introduced recently into modeling: Ly $\alpha$ red-wing opacity (Kowalski \& Saumon 2006), refraction (Kowalski \& Saumon 2004), improved description of ionization equilibrium (Kowalski et al. 2007), and the nonideal dissociation equilibrium of $\mathrm{H}_{2}$ (Kowalski 2006). The models include CIA absorption, and they demonstrate that CIA is expected to create wiggles and bumps in the spectrum up to $3 \mu \mathrm{m}$, and the SEDs of cool WDs are expected to return to normal in the midinfrared. Our Spitzer photometry and spectroscopy do not reveal any wiggles due to molecular absorption. The dashed line in Figure 4 shows the best-fit power law to the infrared portion of the LHS 1126 SED from 1 to $8 \mu \mathrm{m}$. The best-fit power law has an index of -1.99 , identical to a Rayleigh-Jeans tail spectrum. The best-fit power laws for the other 18 stars in our sample have indices from -1.80 for $9000 \mathrm{~K}$ WDs to -1.39 for $5500 \mathrm{~K}$ WDs; LHS 1126 has a unique SED.

\section{DISCUSSION}

Brown dwarfs and massive planets are also expected to suffer from CIA (Borysow et al. 1997). Cushing et al. (2006) and Roellig et al. (2004) obtained mid-infrared spectroscopy of M, $\mathrm{L}$, and T dwarfs; the observed spectra are in good agreement with the model atmosphere calculations, with only a few minor deviations. The only exception to this is 2 MASS $0937+2931$, a T6 dwarf. This object has very blue near-infrared colors, and it shows flux suppression in the $K$ band (Burgasser et al. 2002). Spitzer IRS observations showed that 2MASS $0937+2931$ also shows flux suppression up to $7.5 \mu \mathrm{m}$ compared to typical T dwarfs (Cushing et al. 2006). Burgasser et al. (2002) and Cushing et al. (2006) claimed that this flux suppression may be caused by highpressure (high surface gravity) or low-metallicity atmospheres. The CIA opacities are expected to be stronger in higher pressure environments, and therefore collision-induced $\mathrm{H}_{2} 1-0$ dipole $\mathrm{ab}-$ sorption centered at $2.4 \mu \mathrm{m}$ (Borysow 2002) could explain the $K$-band flux suppression. However, the absorption up to $7.5 \mu \mathrm{m}$ is not predicted by the current CIA opacity calculations. Burgasser et al. (2008) suggest that the observed spectral peculiarities in "blue L dwarfs" can be explained by the presence of thin and/or large-grained condensate clouds.

We find that the cool WD model atmospheres are able to explain the optical and infrared photometry and spectroscopy of all WDs in our sample except LHS 1126, a cool WD only $10 \mathrm{pc}$ away. LHS 1126 displays optical absorption features attributed to the $\mathrm{C}_{2} \mathrm{H}$ molecule. Wickramasinghe et al. (1982) were the first ones to detect a near-infrared flux deficit for LHS 1126. Bergeron et al. $(1994,1997)$ explained this deficit as CIA by molecular hydrogen due to collisions with $\mathrm{He}$ and found the $\log (\mathrm{He} / \mathrm{H})$ ratio to be $\sim 1.86$. Analyzing the UV, optical, and near-IR data, Wolff et al. (2002) found that such a high $\mathrm{H}$ abundance would lead to strong Ly $\alpha$ absorption, and the UV data is better fit with a $\log (\mathrm{He} / \mathrm{H})$ ratio of 5.51 (see Fig. 4). Recently, Kilic et al. (2006) discovered that LHS 1126 also suffers from significant flux deficits at 4.5 and $8 \mu \mathrm{m}$, which are not compatible with the current CIA calculations. Now, we know that this flux deficit is present in all IRAC bands. Our IRS spectrum is also featureless, and it is consistent with a Rayleigh-Jeans tail spectrum. Even though LHS 1126 looks like a cool WD with $T_{\text {eff }}=5400 \mathrm{~K}$ in the optical, its infrared SED is reminiscent of a hotter WD. Our models cannot explain the observed flux deficits in the Spitzer observations.

LHS 1126, like 2MASS 0937+2931, suffers from an unexplained opacity source up to $8 \mu \mathrm{m}$. Both stars are expected to show CIA. This implies that either the current CIA opacity calculations are inadequate or the mid-infrared flux deficits observed in these stars are caused by a graylike absorption process not included in our model atmosphere calculations. The input physics used in WD model atmospheres is mostly based on the ideal gas approximation. The extreme conditions in WD atmospheres require a new look at dense medium effects on the equation of state, opacities, and radiative transfer (see Kowalski 2006).

Unlike brown dwarfs, WD spectra do not suffer from absorption due to water, methane, and ammonia in the mid-infrared; therefore, they are better suited to find and constrain additional opacity sources like CIA. Near/mid-infrared photometry of WDs cooler than $5000 \mathrm{~K}$ will be essential to test these ideas. Such a study is currently underway with the Spitzer Space Telescope infrared camera.

P. M. K. acknowledges partial financial support from RuhrUniversität in Bochum. Support for this work was provided by NASA through awards issued by JPL/Caltech to the University of Texas and The Ohio State University. This work is based in part on observations made with the Spitzer Space Telescope, which is operated by the Jet Propulsion Laboratory, California Institute of Technology, under NASA contract 1407. This publication makes use of data products from the Two Micron All Sky Survey, which is a joint project of the University of Massachusetts and the Infrared Processing and Analysis Center/California Institute of Technology, funded by the National Aeronautics and Space Administration and the National Science Foundation. 
Bergeron, P., Leggett, S. K., \& Ruiz, M. T. 2001, ApJS, 133, 413 Bergeron, P., Ruiz, M. T., \& Leggett, S. K. 1997, ApJS, 108, 339

Bergeron, P., et al. 1994, ApJ, 423, 456

Borysow, A. 2002, A\&A, 390, 779

Borysow, A., et al. 1997, A\&A, 324, 185

Burgasser, A. J., et al. 2002, ApJ, 564, 421 2008, ApJ, 674, 451

Cushing, M. C., et al. 2006, ApJ, 648, 614

Debes, J. H., Sigurdsson, S., \& Hansen, B. 2007, AJ, 134, 1662

Farihi, J., Zuckerman, B., \& Becklin, E. E. 2008, ApJ, 674, 431

Fazio, G. G., et al. 2004, ApJS, 154, 10

Frommhold, L. 1993, Collision-Induced Absorption in Gases (Cambridge: Cambridge Univ. Press)

Gates, E. 2004, ApJ, 612, L129

Hansen, B. M. S. 1998, Nature, 394, 860

Hansen, B. M. S., et al. 2007, ApJ, 671, 380

Harris, H. C., et al. 2001, ApJ, 549, L109 2006, AJ, 131, 571

Houck, J. R., et al. 2004, ApJS, 154, 18
REFERENCES

Jura, M., Farihi, J., Zuckerman, B., \& Becklin, E. E. 2007, AJ, 133, 1927

Kilic, M., von Hippel, T., Mullally, F., Reach, W. T., Kuchner, M. J., Winget, D. E., \& Burrows, A. 2006, ApJ, 642, 1051

Koester, D., \& Wolff, B. 2000, A\&A, 357, 587

Kowalski, P. M. 2006, ApJ, 641, 488

Kowalski, P. M., \& Saumon, D. 2004, ApJ, 607, 970 2006, ApJ, 651, L137

Kowalski, P., et al 2007, Phys. Rev. B. 2007, 76

Liebert, J., Dahn, C. C., \& Monet, D. G. 1988, ApJ, 332, 891

Reach, W. T., Kuchner, M. J., von Hippel, T., Burrows, A., Mullally, F., Kilic, M., \& Winget, D. E. 2005a, ApJ, 635, L161

Reach, W. T., et al. 2005b, PASP, 117, 978

Roellig, T. L., et al. 2004, ApJS, 154, 418

Saumon, D., \& Jacobson, S. B. 1999, ApJ, 511, L107

Tremblay, P.-E., \& Bergeron, P. 2007, ApJ, 657, 1013

Wickramasinghe, D. T., Allen, D. A., \& Bessel, M. S. 1982, MNRAS, 198, 473

Winget, D. E., Hansen, C. J., Liebert, J., van Horn, H. M., Fontaine, G., Nather, R. E., Kepler, S. O., \& Lamb, D. Q. 1987, ApJ, 315, L77

Wolff, B., Koester, D., \& Liebert, J. 2002, A\&A, 385, 995 